\title{
Hyperprogressive Disease in Patients with Non-Small Cell Lung Cancer Treated with Checkpoint Inhibitors: The Role of ${ }^{18}$ F-FDG PET/CT
}

\author{
Angelo Castello ${ }^{1}$, Sabrina Rossi ${ }^{2}$, Emanuela Mazziotti ${ }^{1}$, Luca Toschi $^{2}$, and Egesta Lopci ${ }^{1}$ \\ ${ }^{I}$ Department of Nuclear Medicine, Humanitas Clinical and Research Center, IRCCS, Milan, Italy; and ${ }^{2}$ Department of Oncology and \\ Hematology, Humanitas Clinical and Research Center, IRCCS, Milan, Italy
}

\begin{abstract}
A new pattern of response, so-called hyperprogressive disease (HPD), is emerging during treatment with immune checkpoint inhibitors (ICls). Our aim was to investigate the prevalence of such a phenomenon and to assess its association with clinical variables and metabolic parameters by ${ }^{18} \mathrm{~F}-\mathrm{FDG}$ PET/CT. Methods: Data from 50 patients (34 men and 16 women; median age, $73 \mathrm{y}$ ) with non-small cell lung carcinoma treated with ICls were prospectively collected. All patients underwent contrast-enhanced CT, ${ }^{18} \mathrm{~F}-\mathrm{FDG}$ PET/CT, and complete peripheral blood sampling at baseline before $\mathrm{ICl}$ treatment. HPD was defined according to clinical and radiologic criteria. Because of rapid disease progression or worsening of clinical condition, radiologic response assessment was available for only 46 of the 50 patients. Overall survival (OS) was analyzed using the Kaplan-Meier method and the log-rank test. A Cox proportional hazards regression analysis was used to evaluate factors independently associated with OS. Median follow-up was 12.4 mo (range, 9.7-15.2 mo). Results: We identified the following response categories: 10 cases as complete or partial response, 17 cases as stable disease, 5 cases as progressive disease, and 14 cases as HPD. Among metabolic parameters, we observed a statistically significant association between HPD status and tumor burden, expressed by both TLG $\left(756.1 \mathrm{~cm}^{3}\right.$ for HPD vs. $475.6 \mathrm{~cm}^{3}$ for non-HPD, $P=0.042)$ and MTV (287.3 for HPD vs. 62.1 for nonHPD, $P=0.011$ ). Among clinical variables, 12 of 14 patients $(85.7 \%)$ within the HPD group, compared with 8 of 32 patients $(25 \%)$ in the non-HPD group, had more than 2 metastatic sites $(P<0.001)$. In addition, the derived neutrophil-to-lymphocyte ratio and platelet count were significantly associated with HPD status $(P=0.038$ and $P=0.025$, respectively). Survival analysis showed a median OS of 4 mo for the HPD group, compared with 15 mo for the non-HPD group $(P=0.003)$. Likewise, median OS was significantly different when we considered all the response categories: complete or partial response, stable disease, progressive disease, and HPD $(P=0.001)$. Finally, multivariate analysis identified metabolic tumor volume and derived neutrophil-to-lymphocyte ratio as independent predictors for OS. Conclusion: Our results suggest that the use of ICls might represent a concern in patients with high metabolic tumor burden and inflammatory indices at baseline. However, additional studies are needed.
\end{abstract}

\footnotetext{
Received Oct. 4, 2019; revision accepted Nov. 4, 2019.

For correspondence or reprints contact: Egesta Lopci, Department of Nuclear Medicine, Humanitas Clinical and Research Center, IRCCS, Via Manzoni 56, CAP 20089, Rozzano, Milan, Italy.

E-mail: egesta.lopci@humanitas.it

Published online Dec. 20, 2019.

COPYRIGHT (C 2020 by the Society of Nuclear Medicine and Molecular Imaging.
}

Key Words: non-small cell lung cancer; checkpoint inhibitors; ${ }^{18} \mathrm{~F}-$ FDG PET/CT; hyperprogressive disease

J Nucl Med 2020; 61:821-826

DOI: 10.2967/jnumed.119.237768

C ancer therapy has been positively revolutionized with the introduction of immune checkpoint inhibitors (ICIs) (1). By targeting cytotoxic T-lymphocyte-associated antigen 4 and programmed cell death protein 1 (PD-1) or its ligand, programmed cell death ligand 1 (PD-L1), ICIs restore antitumor T-cell activity and prolong survival in several advanced malignancies, such as melanoma $(2,3)$, non-small cell lung carcinoma (NSCLC) (4), head and neck squamous cell carcinoma (5), renal cell carcinoma $(6,7)$, and Hodgkin lymphomas (8).

Besides the survival advantage, there is growing evidence that treatment with ICIs might, in a subset of patients, have a detrimental effect characterized by a rapid increase in tumor extent with a worse clinical outcome, termed hyperprogressive disease (HPD) (9). Although this phenomenon has been described transversally in different cancer histologic types under immunotherapy, there is no worldwide accepted and clear definition, and the exact underlying pathophysiology remains unknown. As a result, the prevalence of HPD ranges from $4 \%$ to $29 \%$ according to the various criteria adopted in published studies (10-14). To date, weak potential predictive factors for HPD have been identified, all different across various studies. Therefore, it is of critical importance to identify patients with HPD in order to promote early suspension of immunotherapy and switching to another anticancer treatment.

The role of ${ }^{18} \mathrm{~F}-\mathrm{FDG}$ PET/CT for assessing early tumor response in NSCLC patients treated with ICIs is in continuous evolution, as most published studies have evaluated advancedmelanoma patients. However, Lopci et al. (15), reporting a correlation among metabolic parameters and immune markers (e.g., PD-1/PD-L1, CD8, and CD68), suggested ${ }^{18}$ F-FDG PET/CT as a valid tool to explore changes within the tumor microenvironment during ICI treatment. In addition, Kaira et al. (16) demonstrated that metabolic response by ${ }^{18} \mathrm{~F}-\mathrm{FDG}$ could be effective for predicting efficacy and progression-free survival at $1 \mathrm{mo}$ after nivolumab treatment.

On the basis of these findings, the aim of our prospective study was to investigate the prevalence of HPD in patients with NSCLC treated with ICIs and to seek, among clinical and metabolic parameters, potential biomarkers of HPD. 


\section{MATERIALS AND METHODS}

Patients

This study was conducted on patients affected by metastatic or relapsed NSCLC who were referred to our hospital, Humanitas Clinical and Research Center, from December 2015 to May 2019 for treatment with ICIs. The medical records of all consecutive patients $(n=50)$ were analyzed. Prospective data were collected from patients $(n=42)$ adhering to the same diagnostic trial, registered at https://clinicaltrials.gov (NCT03563482), and from other clinical trials for ICIs $(n=8)$. Before the administration of ICIs, all patients underwent ${ }^{18} \mathrm{~F}-\mathrm{FDG}$ PET/CT, brain imaging, and thoracic enhanced multidetector CT. Likewise, baseline white blood cell counts, including absolute neutrophil counts, absolute lymphocyte counts, and platelet counts, were collected. Nivolumab was administered intravenously at a dose of $3 \mathrm{mg} / \mathrm{kg}$ every $2 \mathrm{wk}$, and pembrolizumab was administered at a dose of $200 \mathrm{mg}$ every $3 \mathrm{wk}$.

The study was conducted after approval by the local institutional review board and in accordance with the Declaration of Helsinki and good clinical practice guidelines. Written informed consent was obtained from all patients.

\section{Imaging Protocol}

${ }^{18}$ F-FDG PET/CT. After an approximately 6-h fast, PET/CT images was acquired $60 \mathrm{~min}$ after injection of $250-500 \mathrm{MBq}$ of ${ }^{18} \mathrm{~F}-\mathrm{FDG}$. Two scanners were used, accredited by the EANM Research Ltd. program (19): a Biograph LSO 6 (Siemens), with an integrated 6-slice CT component, and a Discovery PET/CT 690 (GE Healthcare), with an integrated 64-slice CT component. Attenuation-corrected images were obtained with low-dose CT $(120 \mathrm{kV}, 30 \mathrm{~mA})$. An ADW4.6 workstation (GE Healthcare) was used to display images, which were interpreted by 2 experienced nuclear medicine physicians. For the semiquantitative analysis, the threshold for the volumes of interest was set at 0.5 by PETVCAR (GE Healthcare). SUV $\max$ was defined as the value of the highest pixel, and $\mathrm{SUV}_{\text {mean }}$ was defined as being related to the tumor burden. Volumetric parameters for each lesion, such as metabolic tumor volume (MTV) and total lesion glycolysis (TLG = $\mathrm{MTV} \times \mathrm{SUV}_{\text {mean }}$ ), were calculated using an SUV threshold of $42 \%$.

\section{Definition of HPD}

Radiologic evaluation was performed at treatment initiation and every $8 \mathrm{wk}$ thereafter. Tumor response was determined according to RECIST, version 1.1, and immunotherapy-related RECIST (iRECIST) $(17,18)$.

Among the various criteria to define HPD available in the literature (9), we chose those described by Lo Russo et al. (13), which involve NSCLC patients treated with ICIs. In particular, we considered the following parameters: a time to treatment failure of less than 2 mo (time to treatment failure is defined as the time from the start of treatment with ICIs to ICI discontinuation); an increase of at least
$50 \%$ in the sum of target-lesion major diameters between baseline and the first radiologic evaluation; the appearance of at least 2 new lesions in an already-involved organ between baseline and the first radiologic evaluation; spread of the disease to a new organ between baseline and the first radiologic evaluation; and clinical deterioration, with a decrease by at least 2 in Eastern Cooperative Oncology Group performance score during the first 2 mo of treatment. Patients who had at least 3 of these criteria were considered to be experiencing HPD.

\section{Statistical Analysis}

Associations between HPD and categoric or continuous variables were evaluated using the Fisher exact test and the Mann-Whitney test, respectively.

Overall survival (OS) was calculated as the duration between the date of initiation of immunotherapy and the date of death. OS was analyzed using the Kaplan-Meier method and the log-rank test. A Cox proportional-hazards regression analysis was used to evaluate factors independently associated with OS. All statistical analyses were performed using SPSS (version 23.0; IBM) for Windows (Microsoft), and $P$ values of less than 0.05 were considered to be statistically significant.

\section{RESULTS}

Overall, we analyzed 50 patients (34 men and 16 women; median age, $73 \mathrm{y}$ ) with advanced NSCLC treated with ICIs at our hospital. Forty-six patients were evaluable for response, as 4 (8\%) patients stopped ICIs early because of rapid clinical deterioration before the first imaging evaluation at $8 \mathrm{wk}$.

Thirty-one patients (62\%) received nivolumab, 16 (32\%) pembrolizumab, 2 (4\%) a combination of nivolumab and ipilimumab, and only $1(2 \%)$ atezolizumab. The median number of immunotherapy cycles was 6 (range, 1-47). Two patients received only 1 cycle of ICIs, and 4 patients received 2 cycles because of rapid disease progression or worsening of clinical condition.

Median follow-up was 12.4 mo (range, 9.7-15.2 mo). According to iRECIST, we identified the following response categories: complete and partial response (10 patients, $21.7 \%)$, stable disease (17 patients, 37\%), and progressive disease (19 patients, $41.3 \%$ ). Among this last group, 14 patients (30.4\%) fulfilled the criteria for HPD.

\section{Association Between HPD and Clinical-Metabolic Variables}

Among metabolic parameters by ${ }^{18} \mathrm{~F}-\mathrm{FDG}$ PET/CT, we observed a statistically significant association between HPD status and metabolic tumor burden at baseline, expressed by both MTV and TLG. In fact, patients with HPD had higher values of both MTV and TLG than patients without HPD (756.1 vs. $475.6[P=$ $0.011]$ and 287.3 vs. $62.1[P=0.042]$, respectively) (Table 1 ).

TABLE 1

Patient Characteristics and Association Between HPD and Continuous Variables

\begin{tabular}{|c|c|c|c|c|}
\hline Characteristic & All patients $(n=46)$ & Non-HPD $(n=32)$ & $\operatorname{HPD}(n=14)$ & $P$ \\
\hline$S_{\text {SUV }}$ & $15.1(4.9-35.7)$ & $13.8(4.9-25.7)$ & $17.5(5.3-35.7)$ & 0.232 \\
\hline$S U V_{\text {mean }}$ & $6(3.2-10.3)$ & $6.1(3.2-10.3)$ & $5.8(3.3-9)$ & 0.527 \\
\hline MTV & $143.2(2.7-1,772)$ & $62.1(2.7-256.5)$ & $287.3(11.3-1,772)$ & 0.011 \\
\hline TLG & $576.6(12.3-2,504)$ & $475.6(12.3-2,504.1)$ & $756.1(41.8-2,424.5)$ & 0.042 \\
\hline White blood count & 9 (3.8-24.5) & $8.3(4-21.3)$ & $10.2(3.8-24-5)$ & 0.443 \\
\hline Neutrophils & $6.4(1.9-6.9)$ & $5.6(1.9-14.5)$ & $7.8(2-20.9)$ & 0.272 \\
\hline dNLR & $2.7(0.7-12.9)$ & $2.2(0.7-5.5)$ & $3.5(0.9-12.9)$ & 0.038 \\
\hline Platelets & $265.9(118-517)$ & 211 (123-449) & $305(118-517)$ & 0.025 \\
\hline
\end{tabular}


TABLE 2

Patient Characteristics and Association Between HPD and Categoric Variables

\begin{tabular}{|c|c|c|c|c|}
\hline Characteristic & All patients $(n=46)$ & Non-HPD $(n=32)$ & $\operatorname{HPD}(n=14)$ & $P$ \\
\hline Age (y) & & & & $>0.99$ \\
\hline$<73$ & $22(47.8 \%)$ & $15(46.9 \%)$ & $7(50 \%)$ & \\
\hline$\geq 73$ & $24(52.2 \%)$ & $17(53.1 \%)$ & $7(50 \%)$ & \\
\hline Sex & & & & 0.30 \\
\hline Male & $32(69.6 \%)$ & $24(75 \%)$ & $8(57.1 \%)$ & \\
\hline Female & $14(30.4 \%)$ & $8(25 \%)$ & $6(42.9 \%)$ & \\
\hline Smoking status & & & & $>0.99$ \\
\hline Current/former & $41(89.1 \%)$ & $28(87.5 \%)$ & $13(92.9 \%)$ & \\
\hline Never & $5(10.9 \%)$ & $4(12.5 \%)$ & $1(7.1 \%)$ & \\
\hline Previous lines of treatment & & & & 0.71 \\
\hline 0 & $12(26.1 \%)$ & $8(75.0 \%)$ & $4(57.1 \%)$ & \\
\hline$\geq 1$ & $34(73.9 \%)$ & $24(31.3 \%)$ & $10(28.6 \%)$ & \\
\hline Metastatic sites (median) & & & & $<0.001$ \\
\hline$\leq 2$ & $26(69.6 \%)$ & $24(75 \%)$ & $2(14.3 \%)$ & \\
\hline$>2$ & $20(30.4 \%)$ & $8(25 \%)$ & $12(85.7 \%)$ & \\
\hline Histology & & & & $>0.99$ \\
\hline Nonsquamous & $34(73.9 \%)$ & $24(75 \%)$ & $10(71.4 \%)$ & \\
\hline Squamous & $12(26.1 \%)$ & $8(25 \%)$ & $4(28.6 \%)$ & \\
\hline Tumor PD-L1 status & & & & 0.67 \\
\hline Negative & 9 (19.6\%) & 5 (15.6\%) & 4 (28.6\%) & \\
\hline Positive & $16(34.8 \%)$ & 11 (34.4\%) & 5 (35.7\%) & \\
\hline Missing & 21 (45.6\%) & $16(50 \%)$ & 5 (35.7\%) & \\
\hline
\end{tabular}

Furthermore, HPD was significantly associated with more than 2 (median) metastatic sites at baseline: 12 of 14 patients $(85.7 \%)$ within the HPD group compared with 8 of 32 patients (25\%) in the non-HPD group $(P<0.001)$.

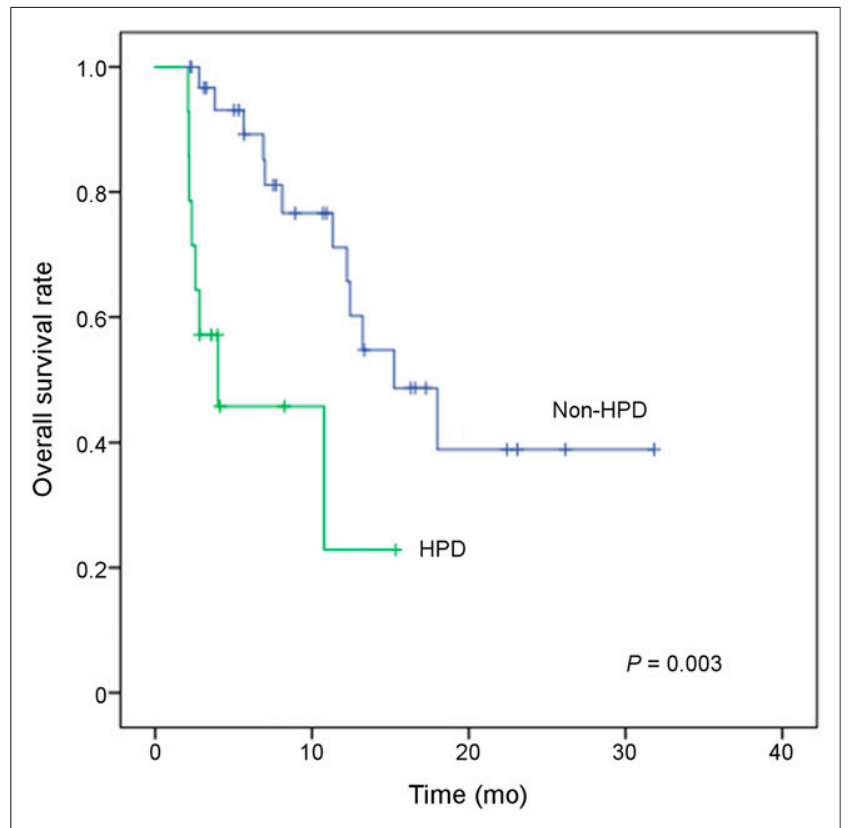

FIGURE 1. OS between HPD and non-HPD patients.
In our cohort, we also analyzed the presence of significant differences between HPD and non-HPD according to inflammatory indices at baseline before ICIs. In particular, the derived

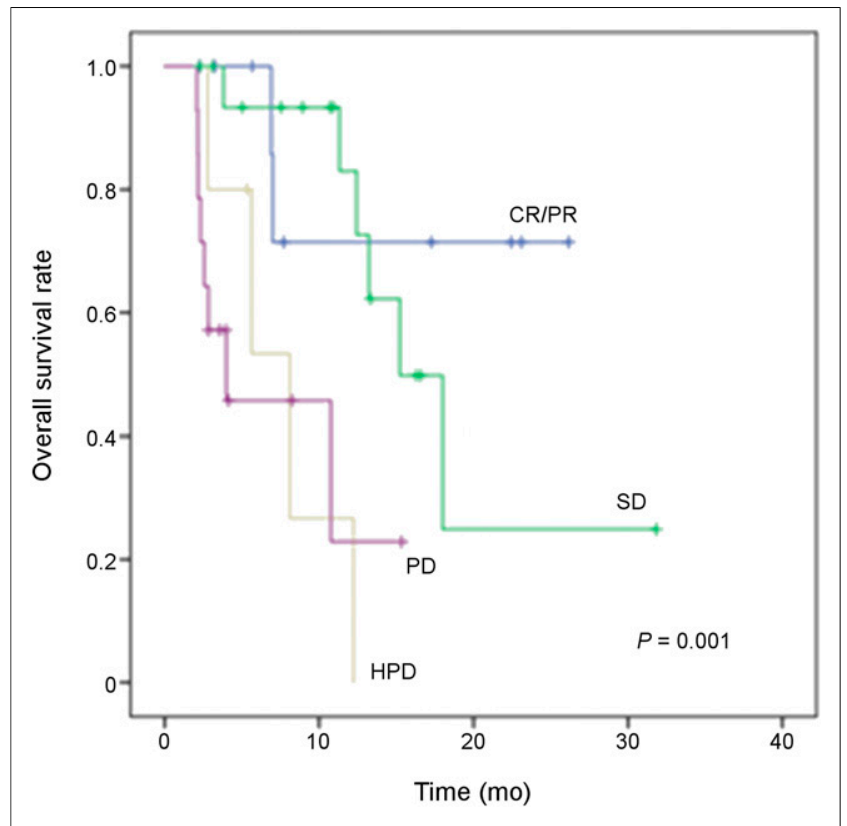

FIGURE 2. OS among response categories according to iRECIST. CR = complete response; $\mathrm{PD}=$ progressive disease; $\mathrm{PR}=$ partial response; $\mathrm{SD}=$ stable disease. 
TABLE 3

Multivariate Analysis of for Overall Survival

\begin{tabular}{lccc}
\hline & \multicolumn{3}{c}{ OS } \\
\cline { 2 - 4 } Parameter & Hazard ratio & $95 \% \mathrm{Cl}$ & $P$ \\
\hline MTV & 1.003 & $1.001-1.005$ & 0.008 \\
dNLR & 1.191 & $1.043-1.359$ & 0.01 \\
\hline
\end{tabular}

neutrophil-to-lymphocyte ratio (dNLR) was significantly associated with HPD status (3.5 in HPD patients vs. 2.2 in non-HPD patients, $P=0.038$ ). Likewise, we found that platelet counts were higher in patients with HPD (305 vs. $211, P=0.025$ ). Among the other clinical variables, no significant differences were observed according to age, sex, smoking history, as well as HPD status was independent from histology. In addition, we examined the influence of previous treatments. Again, we did not observe any association between HPD status and the number of previous therapies. Finally, we were able to assess PD-L1 tumor expression for only 27 patients (54\%) because of insufficient quality or quantity from biopsied material, but we did not observe any difference between HPD patients and other patients, and no association was found between nivolumab or pembrolizumab and HPD status (Table 2).

\section{Association Between HPD and Survival}

Overall, the median OS was 12.4 mo (95\% confidence interval [CI], 9.7-15.2 mo). To evaluate the association between HPD status and prognosis, we performed survival analysis by the Kaplan-Meier method, considering the following categories: complete or partial response, stable disease, non-HPD, and HPD. There was a significant association between HPD and OS, with worse outcome for patients with HPD (median OS, 4.0 mo; 95\% CI, 1-10.6 mo) than for patients with non-HPD (median OS, 15.2 mo; 95\% CI, 9.4-21.1 mo, $P=0.003$ ) (Fig. 1). Moreover, the log-rank test for OS was highly significant among response groups $(P=0.001)$ (Fig. 2).

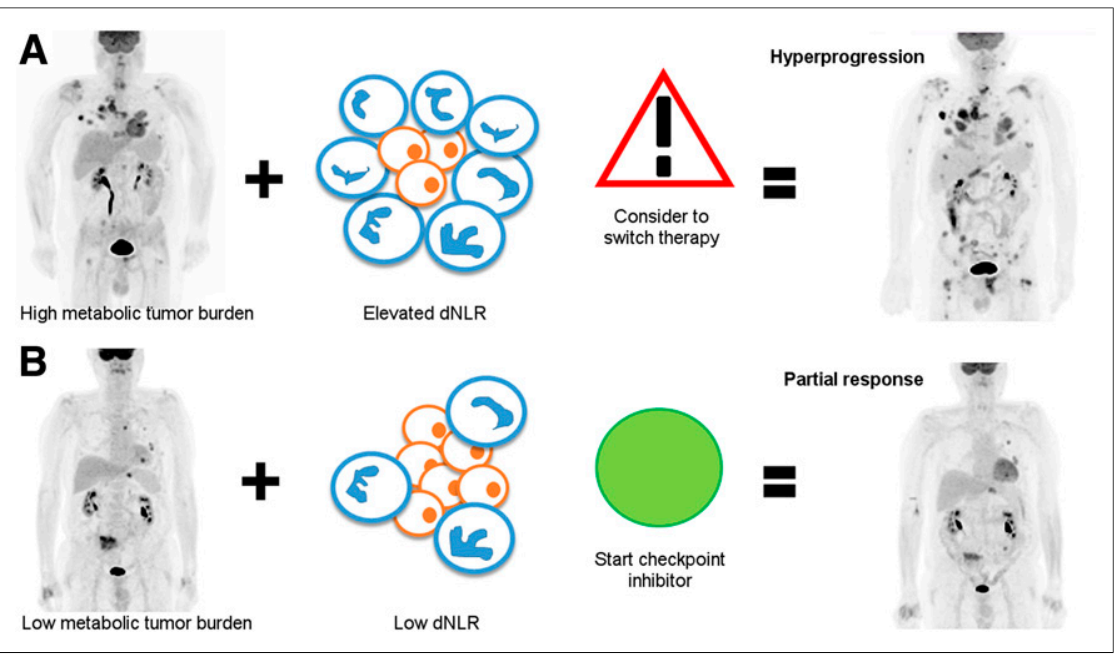

FIGURE 3. Potential algorithm for hyperprogression. (A) Patient with elevated MTV $\left(148 \mathrm{~cm}^{3}\right)$ and dNLR (3.19) at baseline showed hyperprogression after 4 cycles of nivolumab and died approximately 3 mo after ICl therapy started. (B) Patient with low MTV $\left(66 \mathrm{~cm}^{3}\right)$ and dNLR (2.46) experienced partial metabolic response at first restaging and was still alive after approximately $12 \mathrm{mo}$.
Finally, in a multivariate Cox model analysis, we observed that both MTV and dNLR at baseline were strongly associated with OS: MTV had a hazard ratio of 1.003 and a $95 \%$ CI of $1.001-$ $1.005(P=0.008)$, and NLR had a hazard ratio of 1.191 and a 95\% CI of $1.043-1.359(P=0.01)$ (Table 3$)$.

In Figure 3, we suggest a potential algorithm to consider before starting treatment with ICIs.

\section{DISCUSSION}

Although the advent of ICIs in clinical oncology has positively revolutionized the standard of care for patients with NSCLC, oncologists face some challenges. Indeed, on the one hand these new drugs have shown an extraordinary antitumor potential, but on the other hand they may also induce a dramatic tumor surge in a fraction of patients - the so-called hyperprogression.

In our cohort, $30.4 \%$ of evaluable patients $(n=14 / 46)$ with NSCLC experienced HPD during therapy with anti-PD-1 or PDL1. Moreover, we did not consider 4 patients because of clinical deterioration before imaging evaluation; thus, it is likely that the HPD frequency reported here might be an underestimate. The occurrence of HPD also correlated with shorter OS. Previous studies on HPD reported prevalence rates that were quite high, ranging from $9 \%$ to $29 \%$ throughout tumor types, including NSCLC $(10-12,14)$. In all these studies, the tumor growth rate (TGR) before and during ICI treatment was adopted for HPD definition, although with slightly different cutoffs. Nevertheless, such a parameter presupposes radiologic imaging before, at the start of, and during ICI treatment, which is often not the case in clinical practice. Likewise, ICIs are frequently started as first-line therapy, as was also evident in our series (24\% of patients [12/50]); thus, tumor growth rate cannot be computed. Furthermore, being a pure radiologic index, TGR does not take into account clinical condition (e.g., decrease of performance status), leading to a possible underestimation of HPD. Therefore, we adopted stringent criteria, the same as used by Lo Russo et al. (13) in a cohort of NSCLC patients treated with ICIs, which combined both radiologic and clinical parameters. In fact, our HPD frequency was consistent with the paper from the Italian group (13), although further prospective clinical trials are warranted to specifically validate the criteria for HPD diagnosis.

In our series, HPD was significantly associated with a high metabolic tumor burden, expressed by MTV and TLG. To our knowledge, this study was the first to define a metabolic PET-based parameter as a predictor for HPD in the course of ICI therapy. In our preliminary analysis on 27 NSCLC patients, we found that almost all patients with a worse outcome had an $\mathrm{SUV}_{\max }$ of no more than 17.1 or an $\mathrm{SUV}_{\text {mean }}$ of no more than 8.3 (20). The reason for these seemingly contrasting results may lie in the different criteria adopted. In fact, in the preliminary work, we identified patients as "fast progressors or responders" mostly on oncologistbased opinion, instead of using the rigorous criteria of the present work. Furthermore, in our cohort, HPD was significantly associated with a high number of metastatic sites 
before ICI therapy started, as reflects the impact of whole tumor burden on HPD pathophysiology. This finding is in line with a previous publication by Ferrara et al. (11) indicating that HPD was more frequent among NSCLC patients who had more than 2 metastatic sites.

Among clinical variables, dNLR and platelet count were found to be associated with HPD status. These findings are consistent with previous studies, in which a dNLR of greater than 3 was found to negatively influence the survival outcome in NSCLC patients treated with ICIs (21); in addition, thrombocytosis (defined as a platelet count $>450,000 / \mathrm{mm}^{3}$ ) is common in solid tumors and fuels tumor growth, neoangiogenesis, and metastasis (22). Contrary to what was observed by Lo Russo et al. (13), no significant association between HPD and PD-L1 tumor expression was found in our study, although PD-L1 status was not available for 23 patients (46\%). However, our recent paper demonstrated an independent expression between circulating and tissue PD-L1 levels, suggesting a potential prognostic role also for soluble PD-L1 (23). Yet, as reported here, we did not observe any difference in the rate of HPD across the different clinical variables, such as age, sex, tumor type, and performance status. However, the studies by Champiat et al. (10) and Kanjanapan et al. (24) were the only ones in which HPD was associated with age (e.g., $>66$ y) and sex (e.g., female); further studies are needed to confirm these results. In addition, in our study we did not observe any effect related to the number of previous therapies; therefore, the risk that the HPD was related to previous conventional treatments is minimal.

Although hyperprogression is an atypical, but not rare, pattern of response during ICI therapy, the underlying biologic mechanisms are still not completely elucidated. The main hypothesis seems to consider HPD a real immunologic phenomenon that involves both the innate and the adaptive immune system, enhancing growth and cancer development. For instance, Lo Russo et al. (13) suggested that the interaction between ICIs and the Fc receptor on intratumoral macrophages could direct the alternative signaling networks of these cells toward a cancer-promoting function. Likewise, PD-1 blockade increased tumor-infiltrating regulatory $\mathrm{T}$ cells, which suppress antitumor T-cell responses (25). Furthermore, through the production of free radicals that damage the DNA, or by the secretion of growth factors that favorite angiogenesis and tissue remodeling promotion, the immune system plays a fundamental role in cancer development.

Our study had some limitations. The first is lack of a control arm: it would have been interesting to perform a similar analysis on a cohort of patients treated with agents other than ICIs. As a consequence, the immuno-related hypothesis causing hyperprogression cannot be accepted completely. However, Kaplan-Meier curves from the main clinical trials (e.g., CheckMate-026, CheckMate-056, CheckMate-227, and Keynote-042) showed a better OS and progression-free survival in the chemotherapy arm than in the immunotherapy arm in the first 3 mo of treatment, suggesting disease progression or death in the immunotherapy arm earlier than expected (4,26-28). Second, a tumor mutational burden analysis was not available at the time of the current study and should be performed in our future studies. Third, the relatively small number of patients weakens the consistency of our results.

\section{CONCLUSION}

Our results show that HPD might represent a concern for the use of ICIs in patients with high metabolic tumor burden, expressed by either MTV, TLG, or more than 2 metastatic sites, as well as in those with high proinflammatory indices (e.g., elevated platelets and dNLR). Despite the many potential biomarkers that were tested, none reached enough strength because of the different tumor types, small samples, and lack of a standard definition for HPD. Therefore, additional studies are warranted to better understand the molecular basis of HPD in order to identify patients at baseline who are at high risk of developing such an atypical response and allow enough advance time to initiate alternative therapies.

\section{DISCLOSURE}

This study was supported by grant 18923 from Fondazione AIRC (Associazione Italiana per la Ricerca sul Cancro)—an individual grant to Egesta Lopci and fellowships related to the grant to Angelo Castello and Emanuela Mazziotti. No other potential conflict of interest relevant to this article was reported.

\section{KEY POINTS}

QUESTION: IS HPD with ICI real?

PERTINENT FINDINGS: HPD seems a new phenomenon induced by ICI therapy, which accelerates tumor growth. MTV, dNLR, and platelet counts are associated with HPD status. MTV is a prognostic factor for OS.

IMPLICATIONS FOR PATIENT CARE: Clinicians should be aware of HPD in order to carefully monitor disease evolution and, if necessary, to switch to another treatment.

\section{REFERENCES}

1. Ribas A, Wolchok JD. Cancer immunotherapy using checkpoint blockade. Science. 2018;359:1350-1355.

2. Robert C, Long GV, Brady B, et al. Nivolumab in previously untreated melanoma without BRAF mutation. $N$ Engl J Med. 2015;372:320-330.

3. Robert C, Schachter J, Long GV, et al. Pembrolizumab versus ipilimumab in advanced melanoma. N Engl J Med. 2015;372:2521-2532.

4. Borghaei H, Paz-Ares L, Horn L, et al. Nivolumab versus docetaxel in advanced nonsquamous non-small-cell lung cancer. N Engl J Med. 2015;373:16271639.

5. Ferris RL, Blumenschein G, Fayette J, et al. Nivolumab for recurrent squamouscell carcinoma of the head and neck. N Engl J Med. 2016;375:1856-1867.

6. Motzer RJ, Escudier B, McDermott DF, et al. Nivolumab versus everolimus in advanced renal-cell carcinoma. $N$ Engl J Med. 2015;373:1803-1813.

7. Rosenberg JE, Hoffman-Censits J, Powles T, et al. Atezolizumab in patients with locally advanced and metastatic urothelial carcinoma who have progressed following treatment with platinum-based chemotherapy: a single-arm, multicentre, phase 2 trial. Lancet. 2016;387:1909-1920.

8. Ansell SM, Lesokhin AM, Borrello I, et al. PD-1 blockade with nivolumab in relapsed or refractory Hodgkin's lymphoma. N Engl J Med. 2015;372:311-319.

9. Frelaut M, Le Tourneau C, Borcoman E. Hyperprogression under immunotherapy. Int J Mol Sci. 2019;20:E2674.

10. Champiat S, Dercle L, Ammari S, et al. Hyperprogressive disease is a new pattern of progression in cancer patients treated by anti-PD-1/PD-L1. Clin Cancer Res. 2017;23:1920-1928.

11. Ferrara R, Mezquita L, Texier M, et al. Hyperprogressive disease in patients with advanced non-small cell lung cancer treated with PD-1/PD-L1 inhibitors or with single-agent chemotherapy. JAMA Oncol. 2018;4:1543-1552.

12. Saâda-Bouzid E, Defaucheux C, Karabajakian A, et al. Hyperprogression during anti-PD-1/PD-L1 therapy in patients with recurrent and/or metastatic head and neck squamous cell carcinoma. Ann Oncol. 2017;28:1605-1611.

13. Lo Russo G, Moro M, Sommariva M, et al. Antibody-Fc/FcR interaction on macrophages as a mechanism for hyperprogressive disease in non-small cell lung cancer subsequent to PD-1/PD-L1 blockade. Clin Cancer Res. 2019;25:989-999.

14. Kato S, Goodman A, Walavalkar V, Barkauskas DA, Sharabi A, Kurzrock R. Hyper-progressors after immunotherapy: analysis of genomic alterations associated with accelerated growth rate. Clin Cancer Res. 2017;23:4242-4250. 
15. Lopci E, Toschi L, Grizzi F, et al. Correlation of metabolic information on FDGPET with tissue expression of immune markers in patients with non-small cell lung cancer (NSCLC) who are candidates for upfront surgery. Eur J Nucl Med Mol Imaging. 2016;43:1954-1961.

16. Kaira K, Higuchi T, Naruse I, et al. Metabolic activity by ${ }^{18}$ F-FDG-PET/CT is predictive of early response after nivolumab in previously treated NSCLC. Eur J Nucl Med Mol Imaging. 2018;45:56-66.

17. Eisenhauer EA, Therasse $\mathrm{P}$, Bogaerts J, et al. New response evaluation criteria in solid tumours: revised RECIST guideline (version 1. 1). Eur J Cancer. 2009;45: $228-247$.

18. Seymour L, Bogaerts J, Perrone A, et al. iRECIST: guidelines for response criteria for use in trials testing immunotherapeutics. Lancet Oncol. 2017;18:e143-e152.

19. Boellaard R, Delgado-Bolton R, Oyen WJ, et al. FDG PET/CT: EANM procedure guidelines for tumour imaging-version 2.0. Eur J Nucl Med Mol Imaging. 2015;42:328-354.

20. Grizzi F, Castello A, Lopci E. Is it time to change our vision of tumor metabolism prior to immunotherapy? Eur J Nucl Med Mol Imaging. 2018;45:10721075 .

21. Mezquita L, Auclin E, Ferrara R, et al. Association of the Lung Immune Prognostic Index with immune checkpoint inhibitor outcomes in patients with advanced non-small cell lung cancer. JAMA Oncol. 2018;4:351-357.
22. Stone RL, Nick AM, McNeish IA, et al. Paraneoplastic thrombocytosis in ovarian cancer. N Engl J Med. 2012;366:610-618.

23. Grizzi F, Castello A, Qehajaj D, et al. Independent expression of circulating and tissue levels of PD-L1: correlation of clusters with tumor metabolism and outcome in patients with non-small cell lung cancer. Cancer Immunol Immunother. 2019;68:1537-1545.

24. Kanjanapan Y, Day D, Wang L, et al. Hyperprogressive disease in early-phase immunotherapy trials: clinical predictors and association with immune-related toxicities-hyperprogressive disease immunotherapy. Cancer. 2019;125:1341-1349.

25. Kamada T, Togashi Y, Tay C, et al. PD-1+ regulatory T cells amplified by PD-1 blockade promote hyperprogression of cancer. Proc Natl Acad Sci USA. 2019; 116:9999-10008.

26. Carbone DP, Reck M, Paz-Ares L, et al. First line nivolumab in stage IV or recurrent non-small-cell lung cancer. $N$ Engl J Med. 2017;376:2415-2426.

27. Hellmann MD, Ciuleanu T-E, Pluzanski A, et al. Nivolumab plus ipilimumab in Lung cancer with a high tumor mutational burden. $N$ Engl J Med. 2018;378: 2093-2104.

28. Mok TSK, Wu YL, Kudaba I, et al. Pembrolizumab versus chemotherapy for previously untreated, PD-L1-expressing, locally advanced or metastatic nonsmall-cell lung cancer (KEYNOTE-042): a randomised, open-label, controlled, phase 3 trial. Lancet. 2019;393:1819-1830. 